\title{
A Case of Spurious Hypoxemia in an ICU Patient With Leukemic Blast Crisis
}

\author{
Sumit Kapoor ${ }^{\mathrm{a}, \mathrm{c}}$, Jyotsana Thakkar ${ }^{\mathrm{b}}$
}

\begin{abstract}
Arterial blood gas analysis is one of the most common tests performed on patients in the intensive care unit (ICU) to diagnose hypoxemia, a condition with low oxygen tension in the blood $\left(\mathrm{PaO}_{2}\right)$. Hypoxemia can be true or spurious/pseudo. One of the causes of spurious hypoxemia is the presence of metabolically active cells like white blood cells (WBCs) and platelets. We present a case of blast crisis due to acute myelogenous leukemia (AML) presenting with spurious hypoxemia which improved after leukapheresis in our ICU.
\end{abstract}

Keywords: Hypoxemia; Leukemia; Spurious

\section{Introduction}

Arterial blood gas analysis is one of the most common tests performed on patients in the intensive care unit (ICU) to diagnose hypoxemia, a condition with low oxygen tension in the blood $\left(\mathrm{PaO}_{2}\right)[1,2]$. However, $\mathrm{PaO}_{2}$ in the arterial blood sample can be affected by many reasons, one being the presence of metabolically active cells like white blood cells (WBCs) and platelets leading to spurious or pseudo hypoxemia [3]. We present a case of blast crisis due to acute myelogenous leukemia ((AML) presenting with spurious hypoxemia which improved after leukapheresis.

\section{Case Report}

An 85-year-old elderly female with past medical history of

\footnotetext{
Manuscript accepted for publication October 01, 2015

${ }^{a}$ Department of Pulmonary/Critical Care, Baylor College of Medicine, Houston, TX, USA

bDepartment of Nephrology, North Shore Long-Island Jewish Hospital, New York, USA

${ }^{\mathrm{c} C o r r e s p o n d i n g ~ A u t h o r: ~ S u m i t ~ K a p o o r, ~ D e p a r t m e n t ~ o f ~ P u l m o n a r y / C r i t i c a l ~}$ Care, Baylor College of Medicine, Houston, TX, USA.

Email: drkapoorsumit@gmail.com
}

doi: http://dx.doi.org/10.14740/jmc2328w diabetes, hypertension and recent diagnosis of AML was admitted to our ICU with severe metabolic acidosis and acute respiratory failure. She received recent chemotherapy at outside hospital. Her chest X-ray was normal, thereby, ruling out pneumonia or congestive heart failure. Suspicion of acute pulmonary embolism was high with acute hypoxemia in a cancer patient with unimpressive chest X-ray. Transthoracic echocardiography did not show any right heart strain pattern. On arrival, she was clinically in shock, disseminated intravascular coagulation (DIC) and had WBC count of $455,000 / \mu \mathrm{L}$. Her initial arterial blood gas (ABG) on admission after endotracheal intubation showed $\mathrm{pH}$ of $6.64, \mathrm{pCO}_{2}$ of $67 \mathrm{~mm} \mathrm{Hg}$ and $\mathrm{pO}_{2}$ of $29 \mathrm{~mm} \mathrm{Hg}$ on $100 \% \mathrm{FiO}_{2}$ but pulse oximetry read $\mathrm{SpO}_{2}$ of $100 \%$. Her ICU course was complicated by septic shock, multi-system organ failure and refractory metabolic acidosis. Oncology service was consulted, and patient underwent emergent leukapheresis for blast crisis with improvement in WBC count to $140,000 / \mu \mathrm{L}$. Her ABG improved to $\mathrm{pH}$ of $6.89, \mathrm{pCO}_{2}$ of $30 \mathrm{~mm} \mathrm{Hg}$ and $\mathrm{pO}_{2}$ of $351 \mathrm{~mm} \mathrm{Hg}$ with $\mathrm{SpO}_{2}$ of $100 \%$ on pulse oximetry. Her $\mathrm{PO}_{2}$ improved dramatically after leukapheresis and removal of WBC from the blood. Family soon opted for comfort care measures, and she developed asystolic cardiac arrest and died.

\section{Discussion}

Hypoxemia is a serious condition commonly seen in the ICU patients. Underlying physiologic derangements include ventilation/perfusion mismatch, hypoventilation, shunt and abnormality of diffusion capacity [1]. ABG is a common test employed to diagnose hypoxemia. Hypoxemia can be true or spurious. Common clinical conditions leading to hypoxemia in ICU include pneumonia, pulmonary embolism, acute respiratory distress syndrome and congestive heart failure. Pseudo hypoxemia or spurious hypoxemia is an entity where laboratory abnormalities of $\mathrm{PaO}_{2}$ are not a true reflection of patient's bloodstream oxygen tension. Causes of inaccurate measurements of $\mathrm{PaO}_{2}$ include improper sampling, delay in transport with failure to cool the specimen, presence of bubbles in the sample, elevated cell count, blood gas analyzer malfunction or miscalibration or methemoglobinemia $[1,2]$. Spurious hypoxemia has been reported in patients with hyperleukocytosis or thrombocytosis in the setting of hemato- 
logical malignancies $[2,4,5]$. It was first described in 1979 by Fox et al and was coined leukocyte larceny [3]. This is due to the presence of high number of metabolically active WBCs with elevated consumption of dissolved oxygen in the arterial blood samples [6]. The rate of oxygen consumption by WBCs is typically not important clinically in majority of patients with normal blood counts. Spuriously low $\mathrm{PaO}_{2}$ measurements are common when WBC count exceeds $50,000 / \mu \mathrm{L}$ or with severe thrombocytosis. This entity is not very well described in the critical care literature with not many cases reported in ICU setting [1,2]

Patients with hyperleukocytosis in the setting of acute myelogenous or lymphocytic leukemia or blast crisis can develop true or spurious hypoxemia. Causes of true hypoxemia in these patients include sepsis, pulmonary embolism, pulmonary leukostasis, leukemic infiltration, hemorrhage and drug-related toxicities. Inconsistencies between $\mathrm{SpO}_{2}$ with pulse oximetry and $\mathrm{PaO}_{2}$ in the arterial blood can help to differentiate spurious hypoxemia from these causes. In leukocyte larceny, $\mathrm{SpO}_{2}$ is normal and $\mathrm{PaO}_{2}$ is low. Cooling of blood sample on ice and rapid analysis can help reduce the discrepancy.

Pulse oximetry is the most accurate method to assess oxygenation in patients with hyperleukocytosis $[7,8]$. It measures hemoglobin saturation directly and is unaffected by the plasma oxygen tension. Understanding the concept of spurious hypoxemia in patients with hematologic malignancies will help to prevent unnecessary tests like CT scans and ventilation/perfusion scans to rule out pulmonary embolism, minimizing ICU admission of these patients and saving hospital resources and costs.

Our patient did not undergo these expensive tests for the workup of pulmonary embolism as $\mathrm{PaO}_{2}$ improved after leukapheresis.

\section{Conclusion}

ICU physicians should be vigilant about the concept of pseudo/spurious hypoxemia and leukocyte larceny in patients with leukemias and blast crisis. Causes of true hypoxemia still need to be ruled out in the correct clinical setting. Pulse oximetry is the most reliable and easy method to clinically assess oxygenation in patients with leukocyte larceny.

\section{References}

1. Lele AV, Mirski MA, Stevens RD. Spurious hypoxemia. Crit Care Med. 2005;33(8):1854-1856.

2. Horr S, Roberson R, Hollingsworth JW. Pseudohypoxemia in a patient with chronic lymphocytic leukemia. Respir Care. 2013;58(3):e31-33.

3. Fox MJ, Brody JS, Weintraub LR. Leukocyte larceny: a cause of spurious hypoxemia. Am J Med. 1979;67(5):742746.

4. Hess CE, Nichols AB, Hunt WB, Suratt PM. Pseudohypoxemia secondary to leukemia and thrombocytosis. N Engl J Med. 1979;301(7):361-363.

5. Charoenratanakul S, Loasuthi K. Pseudohypoxaemia in a patient with acute leukaemia. Thorax. 1997;52(4):394395.

6. Cline MJ. Metabolism of the circulating leukocyte. Physiol Rev. 1965;45(4):674-720.

7. Sacchetti A, Grynn J, Pope A, Vasso S. Leukocyte larceny: spurious hypoxemia confirmed with pulse oximetry. $\mathrm{J}$ Emerg Med. 1990;8(5):567-569.

8. Weingarten AE, Neuman GG, Segal B, Kushins LG, Fermon C. Pulse oximetry to determine oxygenation in a patient with pseudohypoxemia. Anesth Analg. 1988;67(7):711-712. 